\title{
Circulating MicroRNAs in Plasma of Hepatitis B e Antigen Positive Children Reveal Liver-Specific Target Genes
}

\author{
Thilde Nordmann Winther, ${ }^{1,2}$ Kari Stougaard Jacobsen, ${ }^{1,2}$ Aashiq Hussain Mirza, ${ }^{2}$ \\ Ida Louise Heiberg, ${ }^{1}$ Claus Heiner Bang-Berthelsen, ${ }^{2}$ Flemming Pociot, ${ }^{2}$ and Birthe Hogh ${ }^{1}$ \\ ${ }^{1}$ Department of Paediatrics, Hvidovre Hospital, University of Copenhagen, Kettegaard Allé 30, 2650 Hvidovre, Denmark \\ ${ }^{2}$ Department of Paediatrics and Center for Non-Coding RNA in Technology and Health, Herlev Hospital, University of Copenhagen, \\ Arkaden, 2730 Herlev, Denmark
}

Correspondence should be addressed to Thilde Nordmann Winther; thildewinther@gmail.com

Received 27 August 2014; Accepted 19 November 2014; Published 17 December 2014

Academic Editor: Daisuke Morioka

Copyright (c) 2014 Thilde Nordmann Winther et al. This is an open access article distributed under the Creative Commons Attribution License, which permits unrestricted use, distribution, and reproduction in any medium, provided the original work is properly cited.

\begin{abstract}
Background and Aim. Hepatitis B e antigen positive (HBeAg-positive) children are at high risk of severe complications such as hepatocellular carcinoma and cirrhosis. Liver damage is caused by the host immune response to infected hepatocytes, and we hypothesise that specific microRNAs play a role in this complex interaction between virus and host. The study aimed to identify microRNAs with aberrant plasma expressions in HBeAg-positive children and with liver-specific target genes. Methods. By revisiting our previous screen of microRNA plasma levels in HBeAg-positive and HBeAg-negative children with chronic hepatitis B $(\mathrm{CHB})$ and in healthy controls, candidate microRNAs with aberrant plasma expressions in HBeAg-positive children were identified. MicroRNAs targeting liver-specific genes were selected based on bioinformatics analysis and validated by qRT-PCR using plasma samples from $34 \mathrm{HBeAg}$-positive, $26 \mathrm{HBeAg}$-negative, and 60 healthy control children. Results. Thirteen microRNAs showed aberrant plasma expressions in $\mathrm{HBeAg}$-positive children and targeted liver-specific genes. In particular, three microRNAs were upregulated and one was downregulated in HBeAg-positive children compared to HBeAg-negative and healthy control children, which showed equal levels. Conclusion. The identified microRNAs might impact the progression of CHB in children. Functional studies are warranted, however, to elucidate the microRNAs' role in the immunopathogenesis of childhood CHB.
\end{abstract}

\section{Introduction}

Children with chronic hepatitis B (CHB) have a lifetime risk of developing hepatocellular carcinoma (HCC) up to $25 \%$ and an incidence of cirrhosis of $2-3 \%$ per year $[1,2]$. It is widely accepted that the natural course of $\mathrm{CHB}$ is determined by the host-virus interaction; however, the exact mechanisms responsible for disease progression in children are not fully understood.

Evidence suggests that microRNAs play a role in the complex interaction between the hepatitis $B$ virus and host [3]. Our group recently identified a panel of 16 microRNAs aberrantly expressed in plasma of children with $\mathrm{CHB}$ and suggested a potential role of these microRNAs in the pathogenesis of childhood CHB [4].
Risk of progressive liver disease primarily applies to hepatitis B e antigen positive (HBeAg-positive) children and seroclearance of $\mathrm{HBeAg}$ is a key event in the natural course of disease [5]. Most children who undergo HBeAg seroconversion are defined inactive carriers, with absent or low viral replication, and usually inactive liver histology [5]. Inactive carriers with no signs of cirrhosis at seroconversion do not show disease progression over long-term follow-up (24-29 years) [6-8]. In the present study we hypothesise that microRNAs aberrantly expressed in plasma of HBeAgpositive children might be involved in the development of progressive liver disease.

It is well known that specific host factors in the liver tissue are tightly regulated in patients with CHB [9]. MicroRNAs 
may regulate specific individual targets or function as master regulators of cellular processes, and many microRNAs regulate their targets cooperatively $[10,11]$. Identifying liverspecific target genes for aberrantly expressed microRNAs in $\mathrm{HBeAg}$-positive children may provide better insight in understanding the role of microRNAs in the pathogenesis of childhood CHB.

The present study aimed to identify circulating microRNAs targeting liver-specific genes-specifically in HBeAgpositive children. Our data provide an important resource for future investigations aiming at deciphering the role of specific microRNAs in the pathogenesis of childhood CHB.

\section{Patients and Methods}

2.1. Patients and Healthy Controls. CHB is a notifiable disease in Denmark [12] and the definition of $\mathrm{CHB}$ is hepatitis $\mathrm{B}$ surface antigen (HBsAg) seropositivity for more than six months [13]. Danish children with CHB are mainly adoptees and immigrants from highly endemic countries. During the period from July 2005 to April 2011, all children aged 0-18 years with $\mathrm{CHB}$ who were reported to Statens Serum Institut, Copenhagen, Denmark $(n=202)$, were invited to participate in the study. Parents of 60 children volunteered, and these children were included in the study. The cohort of children with $\mathrm{CHB}$ was previously described [4].

Characteristics of the chronically HBV infected children in brief included mean age: 10.1 years $(S D \pm 3.9$ years, range 0.9-17.3 years); gender: 26 males and 34 females; racial origin: $62 \%(n=37)$ were Asian, $17 \%(n=10)$ were African, and $22 \%$ $(n=13)$ were Caucasian.

Of the 60 children studied, 34 were $\mathrm{HBeAg-positive}$ and 26 were $\mathrm{HBeAg}$-negative (and anti-HBe positive). Mean alanine aminotransferase (ALT) and HBV DNA $( \pm$ SD) were as follows: HBeAg-positive: ALT $47 \pm 28 \mathrm{U} / \mathrm{L}$ and HBV DNA $5 E+08 \pm 1 E+09 \mathrm{IU} / \mathrm{mL}$, and HBeAg-negative: ALT $26 \pm$ $13 \mathrm{U} / \mathrm{L}$ and HBV DNA $9 E+02 \pm 2 E+03 \mathrm{IU} / \mathrm{mL}$. Among HBeAg-positive children, genotypes A, B, C, D, E, and F were identified, and among HBeAg-negative children, genotypes A, B, C, D, and E were identified. Eleven out of $26 \mathrm{HBeAg-}$ negative patients were not successfully genotyped due to low HBV DNA levels (Table 1) [4]. None of the children showed symptoms of their HBV infection, and none of them had received antiviral treatment. The children were followed in accordance with international guidelines [13-15]. All patients were negative for $\mathrm{HIV}$, hepatitis A virus, and hepatitis $\mathrm{C}$ virus.

Furthermore, 60 healthy control children (previously described [4]) were included between August 2010 and August 2011. These children were recruited prior to elective surgery for a hernia or prior to minor orthopaedic surgery at Hvidovre Hospital, University of Copenhagen, Denmark.

Characteristics of the healthy controls in brief are mean age: 7.1 years ( $\mathrm{SD} \pm 3.7$, range $0.7-15.7)$; gender: 33 males and 27 females; racial origin: $15 \%(n=9)$ were Asian, $2 \%(n=1)$ were African, and $83 \%(n=50)$ were Caucasian (Table 1$)$ [4]. The children were tested HBsAg-negative and none of the children had received vaccination against $\mathrm{HBV}$. The children
TABLE 1: Characteristics of children with chronic hepatitis B and of healthy controls [4].

\begin{tabular}{|c|c|c|c|}
\hline & $\begin{array}{r}\text { Chronic } 1 \\
\text { HBeAg pos. }\end{array}$ & $\begin{array}{l}\text { hepatitis B } \\
\text { HBeAg neg. }\end{array}$ & $\begin{array}{l}\text { Healthy } \\
\text { controls }\end{array}$ \\
\hline Number of patients & 34 & 26 & 60 \\
\hline Male & $14(41 \%)$ & $12(46 \%)$ & $33(55 \%)$ \\
\hline Female & $20(59 \%)$ & $14(54 \%)$ & $27(45 \%)$ \\
\hline \multicolumn{4}{|l|}{ Age (years) } \\
\hline Mean & 8.8 & 12.0 & 7.1 \\
\hline $\mathrm{SD}$ & 3.7 & 3.4 & 3.7 \\
\hline \multicolumn{4}{|l|}{ Race } \\
\hline African & 4 & 6 & 1 \\
\hline Asian & 22 & 15 & 9 \\
\hline Caucasian & 8 & 5 & 50 \\
\hline \multicolumn{4}{|c|}{ ALT (U/L ref. value 5-45) } \\
\hline Mean & 46.5 & 25.6 & 14.3 \\
\hline $\mathrm{SD}$ & 27.6 & 12.6 & 5.3 \\
\hline \multicolumn{4}{|l|}{ HBV DNA (IU/mL) } \\
\hline Mean & $5.1 E+08$ & $8.5 E+02$ & NA \\
\hline $\mathrm{SD}$ & $1.2 E+09$ & $2.0 E+03$ & \\
\hline \multicolumn{4}{|l|}{ Genotypes } \\
\hline A & $2(6 \%)$ & $2(8 \%)$ & \\
\hline $\mathrm{B}$ & $11(32 \%)$ & $2(8 \%)$ & \\
\hline $\mathrm{C}$ & $5(15 \%)$ & $2(8 \%)$ & \\
\hline $\mathrm{D}$ & $12(35 \%)$ & $7(27 \%)$ & \\
\hline $\mathrm{E}$ & $3(9 \%)$ & $2(8 \%)$ & \\
\hline $\mathrm{F}$ & $1(3 \%)$ & $\mathbf{0}(0 \%)$ & \\
\hline N/A & $\mathbf{0}(0 \%)$ & $11(42 \%)$ & $60(100 \%)$ \\
\hline
\end{tabular}

ALT: alanine aminotransferase.

N/A: not analysed.

were free from known medical conditions. Blood samples were obtained prior to anaesthesia.

2.2. Ethical Considerations. The study was performed in accordance with the criteria of the Helsinki II Declaration and was approved by the Ethics Committee, Capital Region of Denmark, Reference number H-KF-255584, and the Danish Data Protection Agency, Journal number 2009-41-4193. Parents of all participants provided informed written consent prior to any study procedure.

2.3. Blood Samples. Blood samples were processed as previously described [4]. Briefly, blood samples were collected in EDTA tubes, centrifuged at 2,500 g for 10 minutes at room temperature, separated, aliquoted, and stored at $-80^{\circ} \mathrm{C}$ until further use.

2.4. HBsAg Quantification. HBsAg was quantified as previously described [4], using ARCHITECT HBsAg assay (Abbott, Chicago, IL, USA) according to manufacturer's instructions. 
2.5. Screen of Plasma MicroRNA Levels in Children with $\mathrm{CHB}$ and in Healthy Controls. Recently, our group published an initial screen of plasma microRNA levels in HBeAgpositive, HBeAg-negative, and healthy children [4]. Briefly, microRNA polymerase-chain-reaction (PCR) panels (human panel I and II V2.M/R), miRCURY LNA Universal RT PCR system (Exiqon, Vedbaek, Denmark) were employed to measure plasma levels of 739 human microRNAs in a total of three samples: one sample contained plasma from $10 \mathrm{HBeAg}$-positive children, one sample contained plasma from $10 \mathrm{HBeAg-negative} \mathrm{children,} \mathrm{and} \mathrm{one} \mathrm{sample} \mathrm{contained}$ plasma from 10 healthy controls. Analyses were performed per Exiqon's instructions. According to the manufacturer, the microRNAs covered in the microRNA PCR panels are generally higher expressed and more likely differentially expressed in disease, or more often cited in the literature.

2.6. Selection of Candidate MicroRNAs. For selection of candidate microRNAs we followed stringent criteria and included only data from $\mathrm{HBeAg}$-positive and $\mathrm{HBeAg}-$ negative children. Firstly, based on raw data, microRNAs with $C_{T}$ values above 35 in $\mathrm{HBeAg}$-positive and/or HBeAgnegative children were excluded. Secondly, raw data were normalised using three differentapproaches: global mean, U6, and geometric mean of miR-22-5p, -26a-5p, and 221$3 \mathrm{p}$ as previously described [4]. By using the comparative $C_{T}$ method, the relative expression of each microRNA between the groups was calculated [16]. We focused on microRNAs aberrantly expressed in HBeAg-positive children and identified the top up- and downregulated microRNAs for further analyses.

2.7. Bioinformatics Analysis. Bioinformatics analysis was performed on all candidate microRNAs identified by revisiting our previous screen. Our analysis included retrieval of microRNA target genes with CLIP-Seq (cross-linking immunoprecipitation-high-throughput sequencing) overlap from starBase (sRNA target Base, release 2.1) and subsequently liver-specificity filters were applied on the retrieved microRNA target genes with CLIP-Seq overlap.

2.8. Retrieval of MicroRNA Target Genes with CLIP-Seq Overlap. MicroRNA target genes were retrieved using the starBase, a database that allows a comprehensive exploration of microRNA-target gene interaction maps from CLIP-Seq and Degradome-Seq data [17]. The predicted microRNAtarget gene interactions in starBase are processed from five target prediction tools (TargetScan, PicTar, PITA, miRanda, and RNA22) overlapping with the CLIP-Seq data. Only those target genes that intersected the CLIP-Seq data sets with a biological complexity $\geq 2$ (a measure of reproducibility between biological replicates or experiments to further reduce false positives) were retrieved.

2.9. Liver-Specificity Filter for MicroRNA Target Genes. Human liver-specific genes were retrieved from the TiGER (Tissue Specific-Gene-Expression and Regulation, version 1.0) [18] and TiSGeD (Tissue-Specific Genes Database) [19] databases. The TiGER database encompasses human tissuespecific gene expression profiles or expressed sequence tag (EST) data, cis-regulatory module (CRM) data, and combinatorial gene regulation data for interacting transcription factor (TF) pairs.

TiGER data is based on analyses of 30 human tissues identifying tissue-specific genes (specificity is determined by expression enrichment scores and associated $-\log 10(P$ value)), TFs and CRMs. A gene is defined as tissue-specific if it satisfies the following two conditions: enrichment score $>5$ and $P$ value $<10^{-3.5}$. In case of the TiSGeD, tissuespecific genes are based on biomedical literature and data mining of gene expression profiles for over 100 human tissues. In TiSGeD, relative tissue-specificity of a gene is based on a statistical parameter, SPM, to quantitatively measure the specificity of a gene over tissues. An SPM parameter is a sensitive indicator in quantitative estimation of gene expression patterns. SPM ranges from 0 to 1.0. A value close to 1.0 indicates high tissue specificity of a gene.

From the TiGER database, liver-specific genes were retrieved: 383 genes based on ESTs (309 nonredundant genes); 300 CRM detections (105 nonredundant genes); and $160 \mathrm{TF}$ pairs coregulating in liver (96 nonredundant genes). Two-hundred-and-fifty liver-specific genes were retrieved from the TiSGeD database that included human liver, fetal liver, hepatoma, and HepG2 specific genes. In total, 542 liverspecific genes were retrieved from these resources.

Liver-specific target genes were identified for the microRNAs of interest by comparing all the target genes with four liver-specific gene lists based on TiGER (EST, CRM, TF) and TiSGeD. A target gene was deemed to be liver-specific if it was present in any of the four liver-specific gene lists.

2.10. Validation of Candidate MicroRNAs. Candidate microRNAs targeting liver-specific genes were selected for further validation by quantitative real-time PCR (qRT-PCR). Candidate microRNAs were individually quantified by standard qRT-PCR using total RNA extracted from plasma of 34 HBeAg-positive, $26 \mathrm{HBeAg-negative,} \mathrm{and} 60$ healthy controls.

2.11. RNA Extraction. Total RNA was extracted from plasma using the miRNeasy mini kit (Qiagen, Hilden, Germany) as previously described [4]. RNA was stored at $-80^{\circ} \mathrm{C}$ until further use.

2.12. cDNA Synthesis. cDNA synthesis was performed using the Universal cDNA Synthesis kit (Exiqon, Vedbaek, Denmark) as previously described [4] and cDNA was stored at $-20^{\circ} \mathrm{C}$.

2.13. $q R T-P C R$. qRT-PCRs were performed on Pick \& Mix microRNA PCR panels including predesigned primers for 23 microRNAs: mir-16-2-3p, -28-5p, -30a-5p, -30b-5p, -30c$5 \mathrm{p},-30 \mathrm{e}-3 \mathrm{p},-144-5 \mathrm{p},-148 \mathrm{a}-3 \mathrm{p},-224-3 \mathrm{p},-378 \mathrm{a}-3 \mathrm{p},-548 \mathrm{c}-5 \mathrm{p}$, $-574-3 p,-589-3 p,-605,-636,-639,-654-3 p$, -let-7c, -23a-3p, -451a, 22-5p, -26a-5p, 221-3p (Exiqon, Vedbaek, Denmark). 
Resultant cDNA was diluted $\times 50$ and assayed in $10 \mu \mathrm{L}$ PCR reactions according to the protocol for miRCURY LNA Universal RT microRNA PCR (Exiqon, Vedbaek, Denmark). Negative controls with no template from the reverse transcription reaction were included and profiled like the samples. Thermal cycling was performed on a CFX384 RealTime thermal cycler (Biorad, Hercules, California, USA) in 384 well plates as per Exiqon's instruction. $C_{T}(\max )$ was set to 40 amplification cycles. Analyses were run in triplicate.

2.14. Analysis of qRT-PCR Data. Quality control of the qRTPCR was performed as previously described [4, 20]. Raw data were normalised using the geometric mean of miR$22-3 p,-26 a-5 p$, and $-221-3 p[4,21,22]$ and the comparative $C_{T}$ method was used to analyse the data [16]. Statistical analyses were performed as previously described [4], by using SAS software, version 9.2 (SAS Institute, Cary, NC, USA). Briefly, statistical significances were determined using MannWhitney test and correlation analyses were performed in two steps, both of which used analysis of variance on ranks ( $P$ values from Chi-Squared tests). Due to multiple testing only $P<0.0028$ was regarded as significant (Bonferroni correction).

\section{Results}

3.1. Clinical Parameters. HBsAg was quantified in plasma from 48 out of $60(80 \%)$ children with CHB. Plasma levels of HBsAg were significantly higher in $\mathrm{HBeAg}$-positive children $(1.1 E+08 \pm \mathrm{SD} 9.3 E+07)$ compared to HBeAg-negative children $(1.4 E+07 \pm \mathrm{SD} 1.2 E+07), P<0.001$. It was not possible to measure $\mathrm{HBsAg}$ in plasma from 12 chronically infected children due to lack of sample material $(7 \mathrm{HBeAg}-$ positive and $5 \mathrm{HBeAg-negative).}$

3.2. Selection of Candidate MicroRNAs. We revisited our previous screen on plasma levels of 739 microRNAs in HBeAg-positive, HBeAg-negative, and healthy children [4]. Only data from children with $\mathrm{CHB}$ were included in the present study. Firstly, we included only microRNAs with raw $C_{T}$-values at or below 35 for further downstream analyses. Based on this cut-off, 227 candidate microRNAs were taken for subsequent analysis. Secondly, raw data of the 227 candidate microRNAs were normalised against the global mean; U6; and geometric mean of miR-22-5p, -26a-5p, and -221-3p. The plasma microRNAs levels in HBeAg-positive and HBeAg-negative children were compared and the top up- and downregulated microRNAs were selected. A total of 32 microRNAs were selected as candidate microRNAs. The candidate microRNAs included 14 microRNAs that were previously identified (miR-99a-5p, -100-5p, -122-5p, -122-3p, $-125 b-5 p,-192-5 p,-192-3 p,-193 b-3 p,-194-5 p,-215,-365 a-3 p$, $-455-5$ p, -455-3p, and -855-5p) [4] and 18 "new" candidate microRNAs of which 9 showed higher levels in plasma from $\mathrm{HBeAg}$-positive children compared to $\mathrm{HBeAg}$-negative children (miR-28-5p, -30a-5p, -30b-5p, -30c-5p, -30e-3p, $-148 a-3 p,-378 a-3 p,-574-3 p$, and $-1 e t-7 c)$ and nine showed lower levels (miR-16-2-3p, -144-5p, -224-3p, -548c-5p, -589$3 p,-605,-636,-639$, and 654-3p).

3.3. Identification of Liver-Specific MicroRNA Target Genes. All 32-candidate microRNAs were included in bioinformatics analysis. MicroRNA target genes with CLIP-seq overlap were retrieved from the starBase, 26 microRNAs (miR-28-5p, 30a-5p, -30b-5p, -30c-5p, -30e-3p, -99a-5p, -100-5p, -122-5p, 125b-5p, -148a-3p, -192-5p, -193b-3p, -194-5p, -215, -365a-3p, 378a-3p, -455-5p, -455-3p, -548c-5p, -574-3p, -605, -636, -639, $-654-3 \mathrm{p},-855-5 \mathrm{p}$, and -let-7c) had target genes with CLIP-seq overlap, and a total of 1822 target genes with CLIP-seq overlap were retrieved from the starBase.

A liver-specificity filter was applied on the retrieved microRNA target genes with CLIP-seq-overlap. Sixteen out of 26 microRNAs had liver-specific target genes with CLIPseq overlap (miR-28-5p, -30a-5p, -30e-3p, -125b-5p, -148a3p, -193b-3p, -215, -365a-3p, -378a-3p, -455-5p, -455-3p, $548 c-5 p,-574-3 p,-639,-654-3 p$, and -let-7c), and in total 28 (16 non-redundant) target genes were retrieved. The 16 non-redundant target genes were $A C A D S B, A R I D 1 A, B T G 3$, CEBPG, CPOX, E2F1, FRAT2, GABBR1, GATA6, HOXA9, LEF1, MAZ, PAPD5, SF1, SMAD4, and ZXDB (Table 2).

Furthermore, the remaining ten microRNAs had no liverspecific target genes and did not pass the liver-specificity filter. For these microRNAs, the total numbers of non-liverspecific target genes with CLIP-seq overlap retrieved from the starBase were 1378 (833 nonredundant). Only those target genes predicted by two or more target prediction tools are presented: in total 522 target genes of which 299 are nonredundant (Table S1 in Supplementary Material, available online at http://dx.doi.org/10.1155/2014/791045).

3.4. Validation of Candidate MicroRNAs. Of the 16 candidate microRNAs targeting liver-specific genes, six microRNAs (miR-125b-5p, -193b-3p, -215, -365a-3p, -455-5p, and -455$3 p)$ were validated in our previous study. Interestingly, all these microRNAs were significantly expressed at higher levels in plasma from HBeAg-positive children than in the HBeAg-negative children [4]. The remaining 10 microRNAs were selected for validation in the present study (miR-28$5 p,-30 a-5 p,-30 e-3 p,-148 a-3 p,-378 a-3 p,-548 c-5 p,-574-3 p$, $-639,-654-3 p$, and -let-7c). Individual qRT-PCR analyses of these microRNAs were performed on plasma samples from $34 \mathrm{HBeAg}$-positive, $26 \mathrm{HBeAg}$-negative, and 60 healthy children. Quality control led to the exclusion of samples from four children (one sample from a HBeAg-negative child and three samples from healthy controls) and the exclusion of two microRNAs (miR-148a-3p and -639).

3.5. MicroRNAs Showing Aberrant Plasma Levels in HBeAgPositive Children. We measured the quantity of the eight microRNAs in plasma from $34 \mathrm{HBeAg}$-positive and 25 HBeAg-negative children. Six microRNAs (miR-28-5p, -30a$5 p,-30 e-3 p,-378 a-3 p,-574-3 p$, and -let-7c) were significantly expressed $(P<0.001)$ at higher levels in plasma from HBeAg-positive children than in the HBeAg-negative children. One microRNA (miR-654-3p) was expressed at a lower 
TABLE 2: Liver-specific target genes with CLIP-seq overlap for 16 microRNAs.

\begin{tabular}{|c|c|c|}
\hline MicroRNA (hsa) & Target gene & Gene description \\
\hline $28-5 \mathrm{p}$ & MAZ & MYC-associated zinc finger protein (purine-binding transcription factor) \\
\hline $30 a-5 p$ & ARID1A & AT rich interactive domain $1 \mathrm{~A}$ (SWI-like) \\
\hline $30 e-3 p$ & CEBPG & CCAAT/enhancer binding protein (C/EBP), gamma \\
\hline \multirow{2}{*}{$125 b-5 p$} & FRAT2 & Frequently rearranged in advanced T-cell lymphomas 2 \\
\hline & MAZ & MYC-associated zinc finger protein (purine-binding transcription factor) \\
\hline \multirow{3}{*}{$148 a-3 p$} & ZXDB & Zinc finger, X-linked, duplicated B \\
\hline & ARID1A & AT rich interactive domain $1 \mathrm{~A}$ (SWI-like) \\
\hline & CEBPG & CCAAT/enhancer binding protein (C/EBP), gamma \\
\hline $193 b-3 p$ & MAZ & MYC-associated zinc finger protein (purine-binding transcription factor) \\
\hline 215 & ACADSB & Acyl-CoA dehydrogenase, short/branched chain \\
\hline \multirow{2}{*}{$365 a-3 p$} & HOXA9 & Homeobox A9 \\
\hline & MAZ & MYC-associated zinc finger protein (purine-binding transcription factor) \\
\hline $378 \mathrm{a}-3 \mathrm{p}$ & ARID1A & AT rich interactive domain 1A (SWI-like) \\
\hline $455-5 \mathrm{p}$ & SMAD4 & SMAD family member 4 \\
\hline \multirow{2}{*}{$455-3 p$} & PAPD5 & PAP associated domain containing 5 \\
\hline & MAZ & MYC-associated zinc finger protein (purine-binding transcription factor) \\
\hline \multirow{3}{*}{$548 c-5 p$} & BTG3 & B-cell translocation gene 3 \\
\hline & SF1 & Splicing factor 1 \\
\hline & GATA6 & GATA binding protein 6 \\
\hline \multirow{2}{*}{$574-3 p$} & GABBR1 & Gamma-aminobutyric acid (GABA) B receptor, 1 \\
\hline & E2F1 & E2F transcription factor 1 \\
\hline 639 & LEF1 & Lymphoid enhancer-binding factor 1 \\
\hline \multirow{2}{*}{$654-3 p$} & CPOX & Coproporphyrinogen oxidase \\
\hline & SF1 & Splicing factor 1 \\
\hline \multirow{4}{*}{ let-7c } & HOXA9 & Homeobox A9 \\
\hline & GATA6 & GATA binding protein 6 \\
\hline & CEBPG & CCAAT/enhancer binding protein (C/EBP), gamma \\
\hline & SF1 & Splicing factor 1 \\
\hline
\end{tabular}

level $(P=0.012)$ (Figure 1). Due to multiple testing, however, only $P<0.0028$ was regarded as significant. miR-548c$5 \mathrm{p}$ was expressed at the same level in plasma from $\mathrm{HBeAg-}$ positive and HBeAg-negative children, $P=0.542$ (Figure 1).

To further investigate the microRNA expression patterns of children with $\mathrm{CHB}$ we included data from 57 healthy controls in the statistical analyses. Of the six microRNAs with significantly higher plasma levels in $\mathrm{HBeAg}$-positive children than in HBeAg-negative children, two microRNAs (miR28-5p and miR-30a-5p) showed similar expression levels in plasma from HBeAg-negative and healthy children, $P>$ 0.004, whereas four microRNAs (miR-30e-3p, 378a-3p, 574$3 \mathrm{p}$, and let-7c) showed lowest expression in plasma from healthy controls, $P<0.001$ (Figure 1).

miR-654-3p that showed lower expression in HBeAgpositive children compared to HBeAg-negative children was expressed at same levels in plasma from HBeAg-negative and healthy control children, $P=0.52$ (Figure 1 ).

Levels (fold changes) of the eight microRNAs in plasma from $\mathrm{HBeAg}$-positive and $\mathrm{HBeAg}$-negative children were calculated relative to the plasma levels in healthy controls (Table 3).
3.6. MicroRNA Plasma Levels and Virological Parameters. We examined the correlations between plasma microRNA levels and the virological parameters: viral load and HBsAg quantity. Only children with $\mathrm{CHB}$ were included in these analyses. Firstly, focusing on univariate analyses, we observed strong positive correlations between plasma levels of six microRNAs (miR-28-5p, -30a-5p, -30e-3p, -378a-3p, -574-3p, and - let-7c) and with both viral load and HBsAg quantity, $P<$ 0.001. All six of these microRNAs showed significantly higher plasma levels in HBeAg-positive children when compared to $\mathrm{HBeAg-negative} \mathrm{children.}$

Secondly, multivariate analyses were performed taking into account the effect on plasma microRNA levels of the different virological parameters: HBeAg-status, viral load, and $\mathrm{HBsAg}$ quantity. Interestingly, the strongest correlations were observed between plasma microRNA levels and HBsAg quantity. The correlations were significant for four microRNAs (miR-28-5p, -30e-3p, -574-3p, and -let-7c), $P<0.001$, all of which showed significantly higher levels in HBeAgpositive children when compared to HBeAg-negative children (Figure 2). 

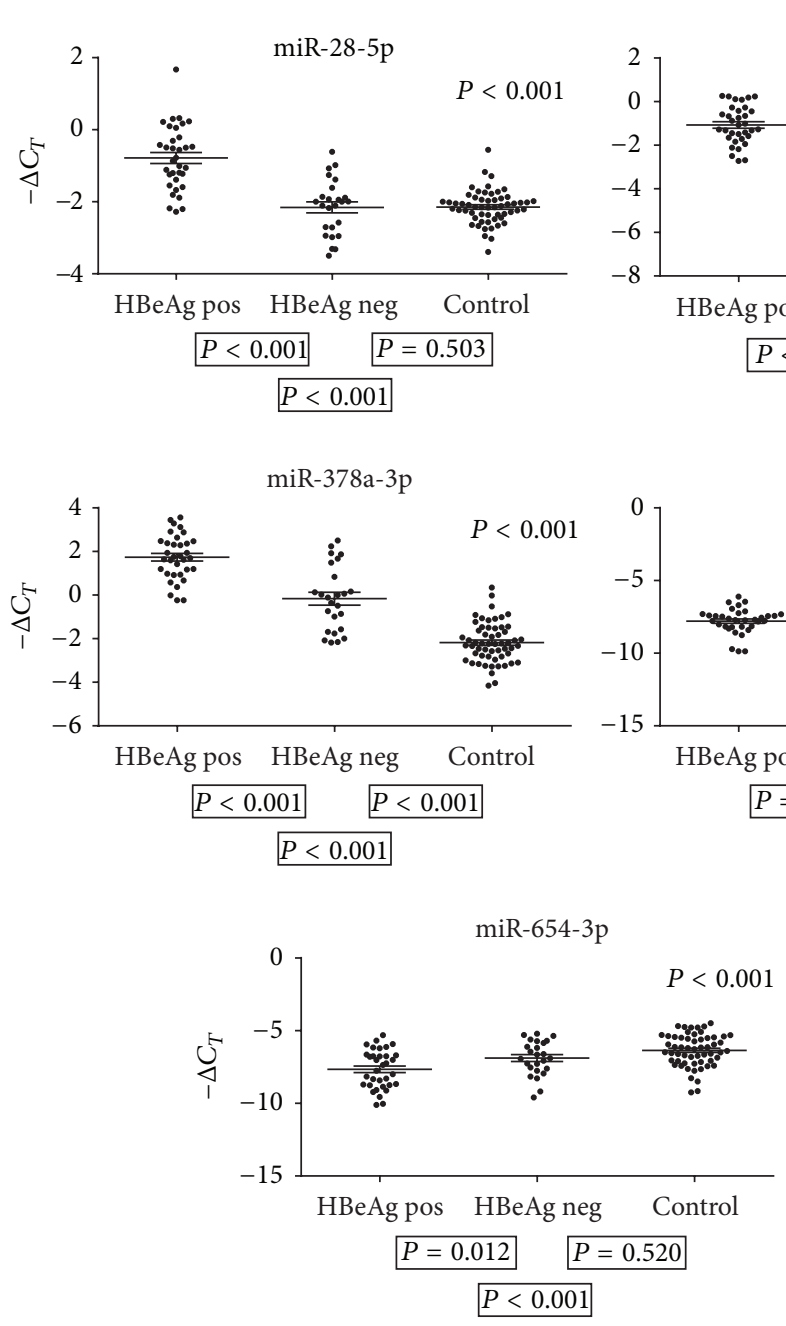
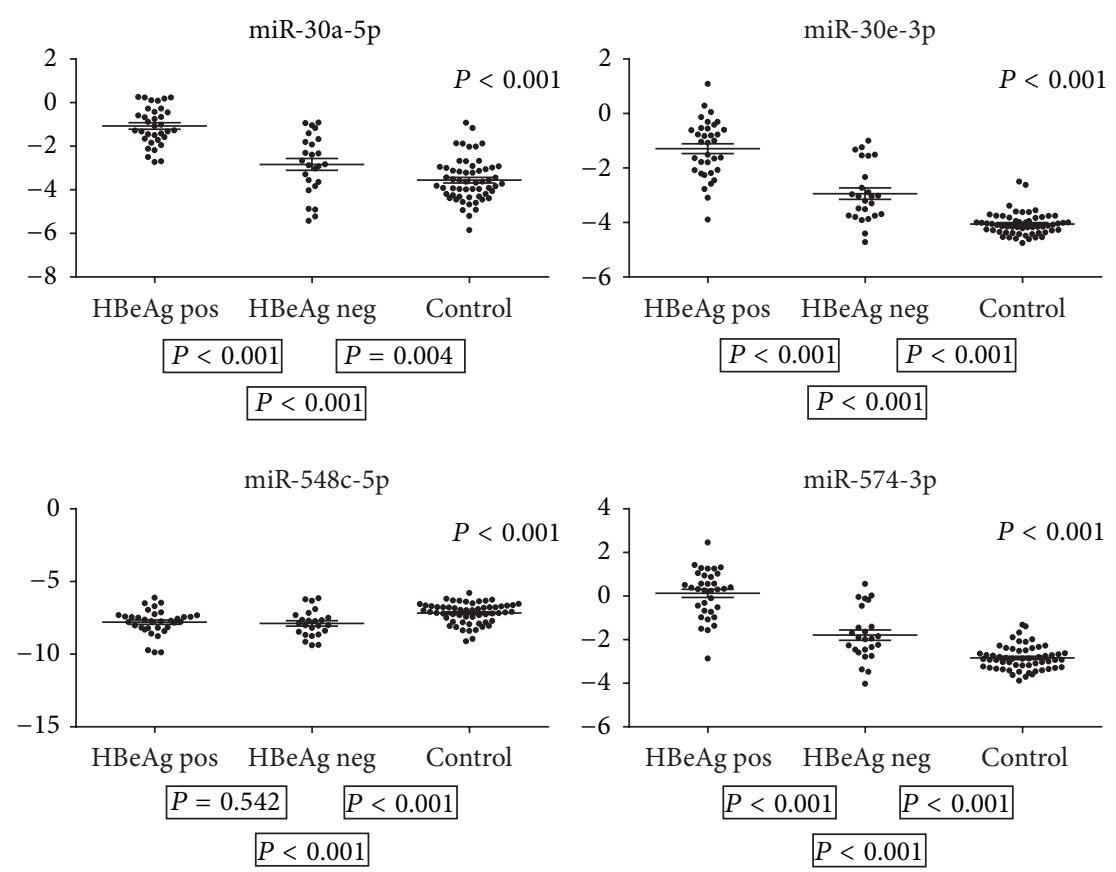

miR-let-7c

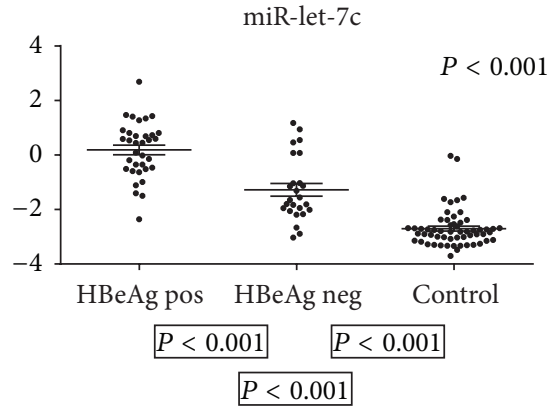

Figure 1: MicroRNA plasma levels in HBeAg-positive, HBeAg-negative, and healthy children. qRT-PCR-data on $34 \mathrm{HBeAg-positive,} 25$ HBeAg-negative, and 57 healthy control children. The $P$ values shown above the individual figures are results of statistical analyses on $\mathrm{HBeAg}-$ positive, $\mathrm{HBeAg}$-negative, and healthy children, and $P$ values shown below left are results on $\mathrm{HBeAg}$-positive versus $\mathrm{HBeAg}$-negative, below centred $\mathrm{HBeAg}$-positive versus healthy controls, and below right HBeAg-negative versus healthy controls. The bars represent geometric means $-\Delta C_{T} \pm$ SEM. Due to multiple testing only $P<0.0028$ was considered significant.

\section{Discussion}

HBeAg-positive children are especially vulnerable to developing HCC and cirrhosis, and we hypothesise that specific microRNAs impact the progression of CHB. This study is the first to identify microRNAs with aberrant plasma expressions specifically in $\mathrm{HBeAg}$-positive children and with liver-specific target genes.

A total of 13 microRNAs showed aberrant plasma expressions in HBeAg-positive children and revealed liverspecific target genes (miR-28-5p, -30a-5p, -30e-3p, -125b-5p, $-193 b-3 p,-215,-365 a-3 p,-378 a-3 p,-455-5 p,-455-3 p,-574-3 p$, $-654-3 p$, and -let-7c). Our bioinformatics approach led to the identification of 21 liver-specific target genes for the 13 microRNAs. Of these, 13 were nonredundant. All target genes retrieved for two or more microRNAs (ARIDIA, CEBPG, HOXA9, and MAZ), and the majority of the remaining target genes (CPOX, E2F1, FRAT2, GATA6, SF1, and SMAD4) are regulators of carcinogenesis, functioning both as promoters and as suppressors, and/or are implicated in the replication of HBV [23-34]. However, three of the target genes, to our knowledge, are not described in relation to cancer or HBV infection (ACADSB, GABBR1, and PAPD5).

For miR-122, known to be highly expressed in liver tissue [35], we could not identify liver-specific target genes due to nonavailability of CLIP-seq data for a number of microRNAs, including mir-122-3p. Additionally, the application of a liver-specificity filter excluded candidate microRNAs with substantial functions in tissues other than the liver.

Of the 13 microRNAs targeting liver-specific genes, seven microRNAs (validated in the present study) were aberrantly expressed in $\mathrm{HBeAg}$-positive children when compared to HBeAg-negative children. Six microRNAs (miR-28-5p, -30a$5 p,-30 e-3 p,-378 a-3 p,-574-3 p$, and -let-7c) showed significantly higher levels in HBeAg-positive children when compared to HBeAg-negative children, and one microRNA 
TABLE 3: MicroRNA plasma levels in children with CHB relative to healthy controls.

\begin{tabular}{|c|c|c|c|c|c|c|}
\hline MicroRNA (hsa) & Sample & Mean Ct & $\mathrm{SD}$ & Fold change & SE & $P$ value \\
\hline \multirow{3}{*}{$28-5 p$} & HBeAg pos. & 28.21 & 1.35 & 3.1 & $2.8-3.5$ & $<0.001$ \\
\hline & HBeAg neg. & 29.89 & 1.80 & 1.0 & $0.9-1.1$ & \\
\hline & Control & 29.39 & 1.34 & 1 & & \\
\hline \multirow{3}{*}{$30 a-5 p$} & HBeAg pos. & 28.50 & 1.22 & 5.6 & $4.9-6.4$ & $<0.001$ \\
\hline & HBeAg neg. & 30.55 & 1.07 & 1.6 & $1.3-2.0$ & \\
\hline & Control & 30.80 & 0.65 & 1 & & \\
\hline \multirow{3}{*}{$30 e-3 p$} & HBeAg pos. & 28.72 & 1.60 & 6.8 & $6.0-7.8$ & $<0.001$ \\
\hline & HBeAg neg. & 30.77 & 1.36 & 2.9 & $2.5-3.3$ & \\
\hline & Control & 31.34 & 1.25 & 1 & & \\
\hline \multirow{3}{*}{$378 a-3 p$} & HBeAg pos. & 25.69 & 1.39 & 15.1 & $13.0-17.5$ & $<0.001$ \\
\hline & HBeAg neg. & 27.88 & 0.93 & 4.0 & $3.3-5.0$ & \\
\hline & Control & 29.43 & 0.71 & 1 & & \\
\hline \multirow{3}{*}{$548 c-5 p$} & HBeAg pos. & 35.17 & 1.57 & 0.7 & $0.6-0.7$ & $<0.001$ \\
\hline & HBeAg neg. & 35.43 & 1.94 & 0.9 & $0.8-1.0$ & \\
\hline & Control & 34.40 & 1.46 & 1 & & \\
\hline \multirow{3}{*}{$574-3 p$} & HBeAg pos. & 27.30 & 1.48 & 7.8 & $6.8-9.0$ & $<0.001$ \\
\hline & HBeAg neg. & 29.51 & 1.22 & 2.1 & $1.7-2.4$ & \\
\hline & Control & 30.07 & 0.98 & 1 & & \\
\hline \multirow{3}{*}{$654-3 p$} & HBeAg pos. & 35.11 & 1.82 & 0.4 & $0.3-0.5$ & $<0.001$ \\
\hline & HBeAg neg. & 34.68 & 2.16 & 0.7 & $0.6-0.8$ & \\
\hline & Control & 33.62 & 1.94 & 1 & & \\
\hline \multirow{3}{*}{ let-7c } & HBeAg pos. & 27.24 & 1.53 & 7.4 & $6.5-8.5$ & $<0.001$ \\
\hline & HBeAg neg. & 29.02 & 1.36 & 2.7 & $2.3-3.2$ & \\
\hline & Control & 29.94 & 1.15 & 1 & & \\
\hline
\end{tabular}

(miR-654-3p) showed lower levels $(P=0.012)$. Additionally, six out of the 13 microRNAs with liver-specific target genes were validated in our previous study. All six microRNAs (miR-125b-5p, -193b-3p, -215, -365a-3p, -455-5p, and -455$3 p)$ [4] showed significantly higher levels in HBeAg-positive children when compared to $\mathrm{HBeAg-negative} \mathrm{children.} \mathrm{Inter-}$ estingly, all 12 microRNAs upregulated in HBeAg-positive children are previously reported aberrantly expressed in adults with different types of cancer [36-44]. Indeed, seven microRNAs (miR-30a-5p, -125b-5p, -193b-3p, -215, -378a-3p, $-574-3 p$, and -let-7c) have previously been associated with HCC $[39-43,45,46]$.

By including data on healthy controls in the analyses we identified two interesting microRNA expression profiles. Firstly, three microRNAs (miR-28-5p, -30a-5p, and -125b-5p) were significantly upregulated in $\mathrm{HBeAg}$-positive children when compared to HBeAg-negative and healthy control children, which showed equal levels. The liver-specific target genes identified for these three microRNAs were ARIDIA, FRAT2, and MAZ. ARID1A encodes an AT-rich interactive domain-containing protein $1 \mathrm{~A}$ (SWI-like). ARID1A is a tumour suppressor and has been found mutated in HBV associated HCC [24, 47]. FRAT2 encodes a GSK-3-binding protein, and the expression of FRAT2 may be upregulated in tumour progression [25]. MAZ gene encodes a MYCassociated zinc finger protein and the expression of MAZ is significantly increased in HCC [26]. Interestingly, the signalling pathways regulated by $A R I D 1 A, F R A T 2$, and $M A Z$ have at least one target in common, namely, c-MYC [48-50], which is known to be overexpressed in mouse HCC [51].

The other interesting microRNA expression profile observed in the present study was for miR-654-3p. miR654-3p was downregulated in HBeAg-positive children when compared to $\mathrm{HBeAg-negative} \mathrm{and} \mathrm{healthy} \mathrm{control} \mathrm{children,}$ which showed equal levels. In line with our finding, miR654-3p has an inhibitory role in the H1N1 Influenza A virus [52] and acts as a tumor suppressor in prostate cancer [53]. Two liver-specific target genes, CPOX and SF1, are targeted by miR-654-3p. CPOX encodes the enzyme Coproporphyrinogen-III oxidase that has a role in the haem and chlorophyll biosynthetic pathways [54] whereas SF1 encodes Splicing Factor 1 that is involved in the formation of the spliceosome complex [55].

We propose that these microRNAs identified with aberrant plasma expressions specifically in HBeAg-positive children and with liver-specific target genes are biomarkers for disease progression and might impact the development of $\mathrm{HCC}$, and perhaps also cirrhosis, in children with $\mathrm{CHB}$. Functional studies are warranted, however, to further elucidate these microRNAs' role in the immunopathogenesis of childhood $\mathrm{CHB}$.

To date few therapies have been licensed to treat $\mathrm{CHB}$ in children, and most are only partially effective [5]. Interestingly, the potential of microRNAs as targets for therapeutic 

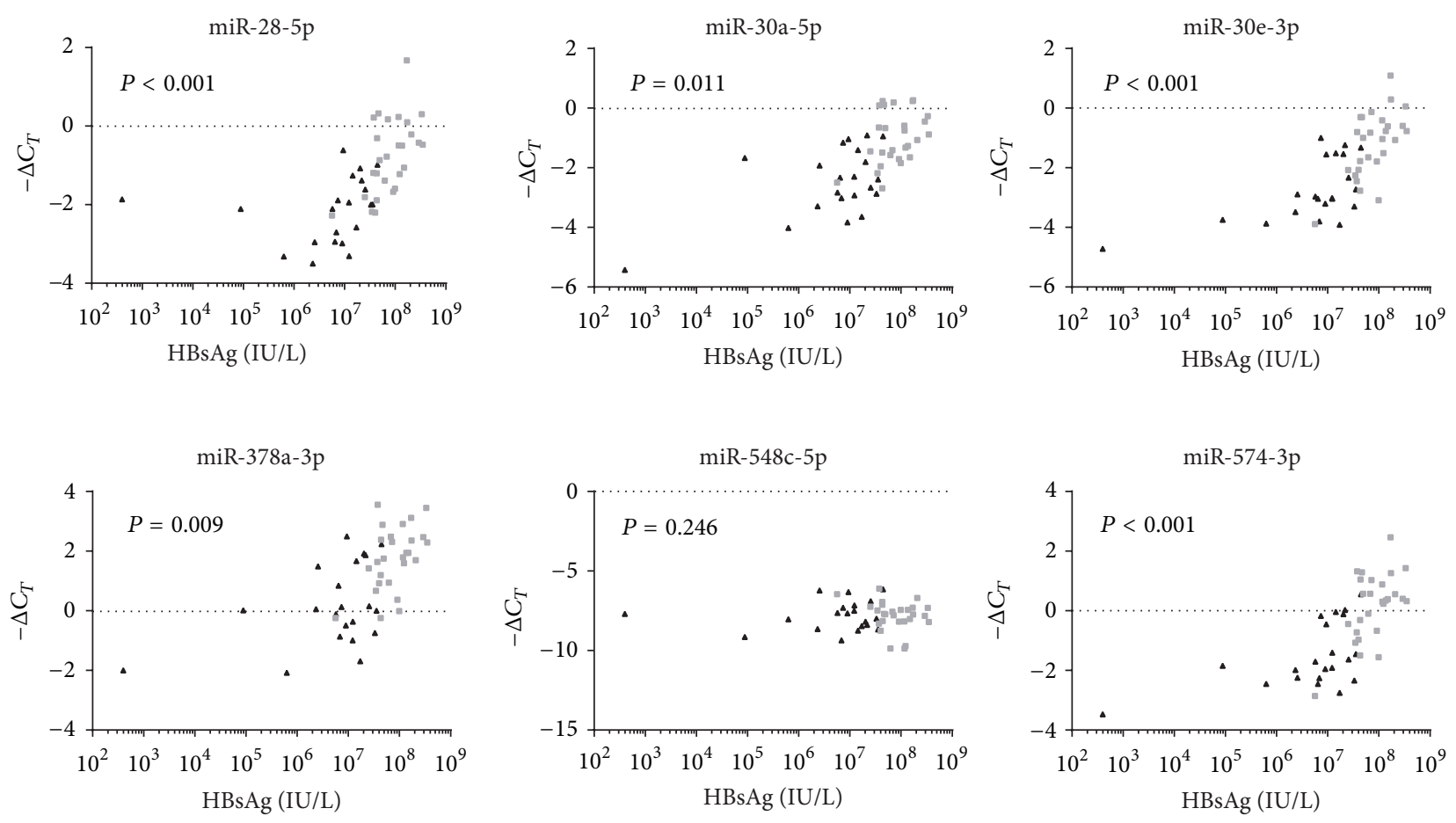

$\operatorname{miR}-654-3 p$

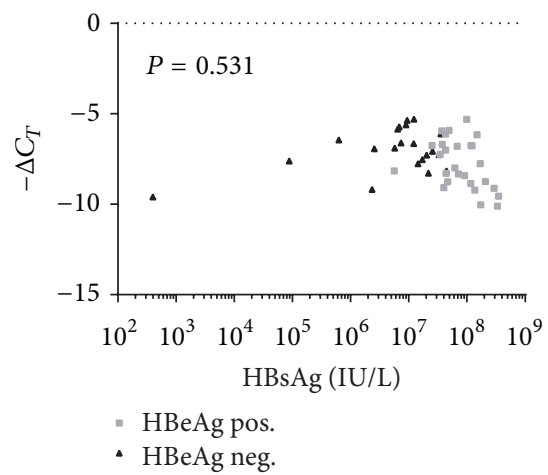

miR-let-7c

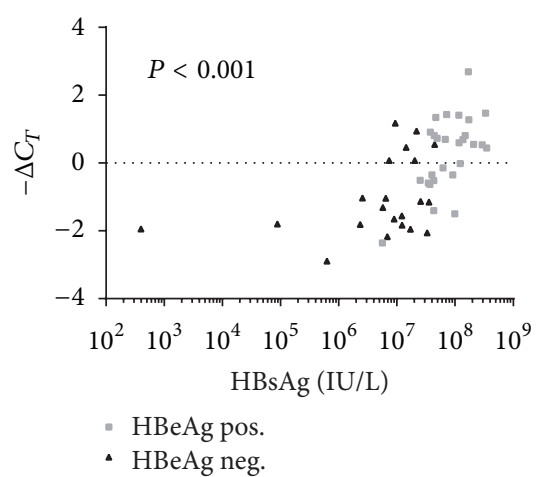

Figure 2: MicroRNA plasma levels and HBsAg quantity. qRT-PCR and HBsAg data on $34 \mathrm{HBeAg}$-positive and $25 \mathrm{HBeAg}$-negative children. $P$ values are based on multivariate analyses. Due to multiple testing only $P<0.0028$ was considered significant.

interventions has shown great promise [11], and the first artificial microRNA antagonist is already in clinical trial [56]. MicroRNAs with a biological function in the immunopathogenesis of childhood $\mathrm{CHB}$ might serve as targets for the development of new antiviral treatment strategies. Whether the microRNAs identified in the present study would have the potential to serve as targets in future therapeutics needs to be investigated.

In conclusion, we identified 13 microRNAs with aberrant plasma expressions of $\mathrm{HBeAg}$-positive children and with liver-specific target genes. In particular, we observed two distinct microRNA expression patterns: three microRNAs were upregulated and one was downregulated in $\mathrm{HBeAg-}$ positive children compared to HBeAg-negative and healthy control children, which showed equal levels. Five liverspecific target genes were identified for the four microRNAs.
The microRNAs might be biomarkers for disease progression in children with $\mathrm{CHB}$. Further studies are needed to elucidate the microRNAs' role in childhood CHB, hopefully leading to the identification of future therapeutic targets and to enhanced management of childhood CHB.

\section{Conflict of Interests}

The authors declare that there is no conflict of interests regarding the publication of this paper.

\section{Acknowledgments}

The authors would like to thank all of the participating children and their parents. They are grateful for the collaboration with the participating hospitals and the staff. A special 
thanks goes to Dr. Thomas Hoffmann, Nina Lambertus, and Lena Vang, Department of Paediatrics, Hvidovre Hospital, University of Copenhagen, Copenhagen, Denmark.

\section{References}

[1] M.-W. Yu, H.-C. Chang, Y.-F. Liaw et al., "Familial risk of hepatocellular carcinoma among chronic hepatitis B carriers and their relatives," Journal of the National Cancer Institute, vol. 92, no. 14, pp. 1159-1164, 2000.

[2] Z. Luo, L. Li, and B. Ruan, "Impact of the implementation of a vaccination strategy on hepatitis B virus infections in China over a 20-year period," International Journal of Infectious Diseases, vol. 16, no. 2, pp. e82-e88, 2012.

[3] Y.-F. Wei, G.-Y. Cui, P. Ye, J.-N. Chen, and H.-Y. Diao, "MicroRNAs may solve the mystery of chronic hepatitis B virus infection," World Journal of Gastroenterology, vol. 19, no. 30, pp. 4867-4876, 2013.

[4] T. N. Winther, C. H. Bang-Berthelsen, I. L. Heiberg, F. Pociot, and B. Hogh, "Differential plasma microRNA profiles in HBeAg positive and $\mathrm{HBeAg}$ negative children with chronic hepatitis B," PLoS ONE, vol. 8, no. 3, Article ID e58236, 2013.

[5] E. M. Sokal, M. Paganelli, S. Wirth et al., "Management of chronic hepatitis B in childhood: ESPGHAN clinical practice guidelines: consensus of an expert panel on behalf of the European Society of Pediatric Gastroenterology, Hepatology and Nutrition," Journal of Hepatology, vol. 59, no. 4, pp. 814-829, 2013.

[6] F. Bortolotti, M. Guido, S. Bartolacci et al., "Chronic hepatitis B in children after e antigen seroclearance: final report of a 29year longitudinal study," Hepatology, vol. 43, no. 3, pp. 556-562, 2006.

[7] R. Iorio, A. Giannattasio, F. Cirillo, L. D'Alessandro, and A. Vegnente, "Long-term outcome in children with chronic hepatitis B: a 24-year observation period," Clinical Infectious Diseases, vol. 45, no. 8, pp. 943-949, 2007.

[8] Y.-H. Ni, M.-H. Chang, P.-J. Chen et al., "Viremia profiles in children with chronic hepatitis B virus infection and spontaneous e antigen seroconversion," Gastroenterology, vol. 132, no. 7, pp. 2340-2345, 2007.

[9] Y. Chen, A. Shen, P. J. Rider et al., "A liver-specific microRNA binds to a highly conserved RNA sequence of hepatitis B virus and negatively regulates viral gene expression and replication," The FASEB Journal, vol. 25, no. 12, pp. 4511-4521, 2011.

[10] R. M. O'Connell, D. S. Rao, A. A. Chaudhuri, and D. Baltimore, "Physiological and pathological roles for microRNAs in the immune system," Nature Reviews Immunology, vol. 10, no. 2, pp. 111-122, 2010.

[11] M. Esteller, "Non-coding RNAs in human disease," Nature Reviews Genetics, vol. 12, no. 12, pp. 861-874, 2011.

[12] Statens Serum Institut (SSI), Mandatory Notification Systems, SSI, Copenhagen, Denmark, 2013, http://www.ssi.dk/English/ HealthdataandICT/Surveillance\%20in\%20Denmark/Mandatory\%20notification $\% 20$ systems.aspx.

[13] "EASL clinical practice guidelines: management of chronic hepatitis B virus infection," Journal of Hepatology, vol. 57, no. 1, pp. 167-185, 2012.

[14] B. A. Haber, J. M. Block, M. M. Jonas et al., "Recommendations for screening, monitoring, and referral of pediatric chronic hepatitis B," Pediatrics, vol. 124, no. 5, pp. e1007-e1013, 2009.
[15] A. S. F. Lok and B. J. McMahon, "Chronic hepatitis B: update of recommendations," Hepatology, vol. 39, no. 3, pp. 857-861, 2004.

[16] K. J. Livak and T. D. Schmittgen, "Analysis of relative gene expression data using real-time quantitative PCR and the 2$\Delta \Delta$ CT method," Methods, vol. 25, no. 4, pp. 402-408, 2001.

[17] J.-H. Yang, J.-H. Li, P. Shao, H. Zhou, Y.-Q. Chen, and L.H. Qu, "StarBase: a database for exploring microRNA-mRNA interaction maps from Argonaute CLIP-Seq and DegradomeSeq data," Nucleic Acids Research, vol. 39, no. 1, pp. D202-D209, 2011.

[18] X. Liu, X. Yu, D. J. Zack, H. Zhu, and J. Qian, “TiGER: a database for tissue-specific gene expression and regulation," BMC Bioinformatics, vol. 9, article 271, 2008.

[19] S.-J. Xiao, C. Zhang, Q. Zou, and Z.-L. Ji, “TiSGeD: a database for tissue-specific genes," Bioinformatics, vol. 26, no. 9, Article ID btq109, pp. 1273-1275, 2010.

[20] T. N. Winther, I. L. Heiberg, C. H. Bang-Berthelsen, F. Pociot, and B. Hogh, "Hepatitis B surface antigen quantity positively correlates with plasma levels of microRNAs differentially expressed in immunological phases of chronic hepatitis B in children," PLoS ONE, vol. 8, no. 11, Article ID e80384, 2013.

[21] H.-T. Zhu, Q.-Z. Dong, G. Wang et al., "Identification of suitable reference genes for qRT-PCR analysis of circulating microRNAs in hepatitis B virus-infected patients," Molecular Biotechnology, vol. 50, no. 1, pp. 49-56, 2012.

[22] J. Vandesompele, K. De Preter, F. Pattyn et al., "Accurate normalization of real-time quantitative RT-PCR data by geometric averaging of multiple internal control genes," Genome Biology, vol. 3, no. 7, 2002.

[23] G. Amaddeo, C. Guichard, S. Imbeaud, and J. Zucman-Rossi, "Next-generation sequencing identified new oncogenes and tumor suppressor genes in human hepatic tumors," OncoImmunology, vol. 1, no. 9, pp. 1612-1613, 2012.

[24] J. Huang, Q. Deng, Q. Wang et al., "Exome sequencing of hepatitis B virus-associated hepatocellular carcinoma," Nature Genetics, vol. 44, no. 10, pp. 1117-1121, 2012.

[25] T. Saitoh, J. Moriwaki, J. Koike et al., "Molecular cloning and characterization of FRAT2, encoding a positive regulator of the WNT signaling pathway," Biochemical and Biophysical Research Communications, vol. 281, no. 3, pp. 815-820, 2001.

[26] J. Dudas, T. Mansuroglu, F. Moriconi et al., "Altered regulation of Proxl-gene-expression in liver tumors," BMC Cancer, vol. 8, article 92, 2008.

[27] Q. Feng, J. E. Stern, S. E. Hawes, H. Lu, M. Jiang, and N. B. Kiviat, "DNA methylation changes in normal liver tissues and hepatocellular carcinoma with different viral infection," Experimental and Molecular Pathology, vol. 88, no. 2, pp. 287292, 2010.

[28] N. Trehanpati, S. Shrivastav, B. Shivakumar et al., "Analysis of Notch and TGF- $\beta$ signaling expression in different stages of disease progression during hepatitis B virus infection," Clinical and Translational Gastroenterology, vol. 3, article e23, 2012.

[29] L. Yao, F.-J. Li, Z.-Q. Tang, S. Gao, and D.-Q. Wu, "Smad4 expression in hepatocellular carcinoma differs by hepatitis status," Asian Pacific Journal of Cancer Prevention, vol. 13, no. 4, pp. 1297-1303, 2012.

[30] E. A. Conner, E. R. Lemmer, M. Omori, P. J. Wirth, V. M. Factor, and S. S. Thorgeirsson, "Dual functions of E2F-1 in a transgenic mouse model of liver carcinogenesis," Oncogene, vol. 19, no. 44, pp. 5054-5062, 2000. 
[31] K. Takahashi, N. Ikeda, N. Nonoguchi et al., "Enhanced expression of coproporphyrinogen oxidase in malignant brain tumors: CPOX expression and 5-ALA-induced fluorescence," NeuroOncology, vol. 13, no. 11, pp. 1234-1243, 2011.

[32] F. Shen, J. Li, W. Cai et al., "GATA6 predicts prognosis and hepatic metastasis of colorectal cancer," Oncology Reports, vol. 30, no. 3, pp. 1355-1361, 2013.

[33] T. Heise, G. Sommer, K. Reumann, I. Meyer, H. Will, and H. Schaal, "The hepatitis B virus PRE contains a splicing regulatory element," Nucleic Acids Research, vol. 34, no. 1, pp. 353-363, 2006.

[34] Y.-H. Ou, P.-H. Chung, F.-F. Hsu, T.-P. Sun, W.-Y. Chang, and S.-Y. Shieh, "The candidate tumor suppressor BTG3 is a transcriptional target of p53 that inhibits E2F1," The EMBO Journal, vol. 26, no. 17, pp. 3968-3980, 2007.

[35] P. Landgraf, M. Rusu, R. Sheridan et al., "A mammalian microRNA expression atlas based on small RNA library sequencing," Cell, vol. 129, no. 7, pp. 1401-1414, 2007.

[36] Z. Li, X. Gu, Y. Fang, J. Xiang, and Z. Chen, "microRNA expression profiles in human colorectal cancers with brain metastases," Oncology Letters, vol. 3, no. 2, pp. 346-350, 2012.

[37] H. Xie, L. Lee, S. Caramuta et al., "MicroRNA expression patterns related to merkel cell polyomavirus infection in human Merkel cell carcinoma," The Journal of Investigative Dermatology, vol. 134, no. 2, pp. 507-517, 2014.

[38] A. Markou, I. Sourvinou, P. A. Vorkas, G. M. Yousef, and E. Lianidou, "Clinical evaluation of microRNA expression profiling in non small cell lung cancer," Lung Cancer, vol. 81, no. 3, pp. 388-396, 2013.

[39] L. Li, Z. Guo, J. Wang, Y. Mao, and Q. Gao, "Serum miR-18a: a potential marker for hepatitis B virus-related hepatocellular carcinoma screening," Digestive Diseases and Sciences, vol. 57, no. 11, pp. 2910-2916, 2012.

[40] J. Gui, Y. Tian, X. Wen et al., "Serum microRNA characterization identifies miR-885-5p as a potential marker for detecting liver pathologies," Clinical Science, vol. 120, no. 5, pp. 183-193, 2011.

[41] X.-M. Zhu, L.-J. Wu, J. Xu, R. Yang, and F.-S. Wu, "Let-7c MicroRNA expression and clinical significance in hepatocellular carcinoma," Journal of International Medical Research, vol. 39, no. 6, pp. 2323-2329, 2011.

[42] H.-Y. Jia, Y.-X. Wang, W.-T. Yan et al., "MicroRNA-125b functions as a tumor suppressor in hepatocellular carcinoma cells," International Journal of Molecular Sciences, vol. 13, no. 7, pp. 8762-8774, 2012.

[43] C. Xu, S. Liu, H. Fu et al., "MicroRNA-193b regulates proliferation, migration and invasion in human hepatocellular carcinoma cells," European Journal of Cancer, vol. 46, no. 15, pp. 2828-2836, 2010.

[44] S.-M. Kang, H.-J. Lee, and J.-Y. Cho, "MicroRNA-365 regulates NKX2-1, a key mediator of lung cancer," Cancer Letters, vol. 335, no. 2, pp. 487-494, 2013.

[45] S. Shimizu, T. Takehara, H. Hikita et al., "The let-7 family of microRNAs inhibits Bcl-xL expression and potentiates sorafenib-induced apoptosis in human hepatocellular carcinoma," Journal of Hepatology, vol. 52, no. 5, pp. 698-704, 2010.

[46] L. Zeng, J. Yu, T. Huang et al., "Differential combinatorial regulatory network analysis related to venous metastasis of hepatocellular carcinoma," BMC Genomics, vol. 13, supplement 8, article S14, 2012.
[47] A. Fujimoto, Y. Totoki, T. Abe et al., "Whole-genome sequencing of liver cancers identifies etiological influences on mutation patterns and recurrent mutations in chromatin regulators," Nature Genetics, vol. 44, no. 7, pp. 760-764, 2012.

[48] S. A. Bossone, C. Asselin, A. J. Patel, and K. B. Marcu, "MAZ, a zinc finger protein, binds to c-MYC and $\mathrm{C} 2$ gene sequences regulating transcriptional initiation and termination," Proceedings of the National Academy of Sciences of the United States of America, vol. 89, no. 16, pp. 7452-7456, 1992.

[49] N. G. Nagl Jr., D. R. Zweitzig, B. Thimmapaya, G. R. Beck Jr., and E. Moran, "The $c-m y c$ gene is a direct target of mammalian SWI/SNF-related complexes during differentiation-associated cell cycle arrest," Cancer Research, vol. 66, no. 3, pp. 1289-1293, 2006.

[50] M. Bechard, R. Trost, A. M. Singh, and S. Dalton, "Frat is a phosphatidylinositol 3-kinase/akt-regulated determinant of glycogen synthase kinase $3 \beta$ subcellular localization in pluripotent cells," Molecular and Cellular Biology, vol. 32, no. 2, pp. 288296, 2012.

[51] C. Coulouarn, L. E. Gomez-Quiroz, J.-S. Lee et al., "Oncogenespecific gene expression signatures at preneoplastic stage in mice define distinct mechanisms of hepatocarcinogenesis," Hepatology, vol. 44, no. 4, pp. 1003-1011, 2006.

[52] L. Song, H. Liu, S. Gao, W. Jiang, and W. Huang, "Cellular microRNAs inhibit replication of the H1N1 influenza A virus in infected cells," Journal of Virology, vol. 84, no. 17, pp. 8849-8860, 2010.

[53] A. Formosa, E. K. Markert, A. M. Lena et al., "MicroRNAs, miR154, miR-299-5p, miR-376a, miR-376c, miR-377, miR-381, miR487b, miR-485-3p, miR-495 and miR-654-3p, mapped to the $14 \mathrm{q} 32.31$ locus, regulate proliferation, apoptosis, migration and invasion in metastatic prostate cancer cells," Oncogene, vol. 33, pp. 5173-5182, 2014.

[54] S. Taketani, T. Furukawa, and K. Furuyama, "Expression of coproporphyrinogen oxidase and synthesis of hemoglobin in human erythroleukemia K562 cells," European Journal of Biochemistry/FEBS, vol. 268, no. 6, pp. 1705-1711, 2001.

[55] M. Corioni, N. Antih, G. Tanackovic, M. Zavolan, and A. Krämer, "Analysis of in situ pre-mRNA targets of human splicing factor SF1 reveals a function in alternative splicing," Nucleic Acids Research, vol. 39, no. 5, pp. 1868-1879, 2011.

[56] H. L. Janssen, H. W. Reesink, E. J. Lawitz et al., "Treatment of HCV infection by targeting microRNA," The New England Journal of Medicine, vol. 368, no. 18, pp. 1685-1694, 2013. 


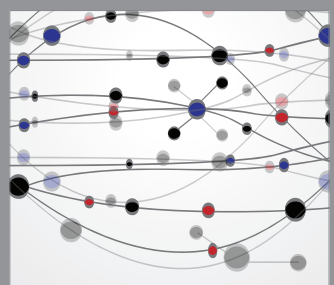

The Scientific World Journal
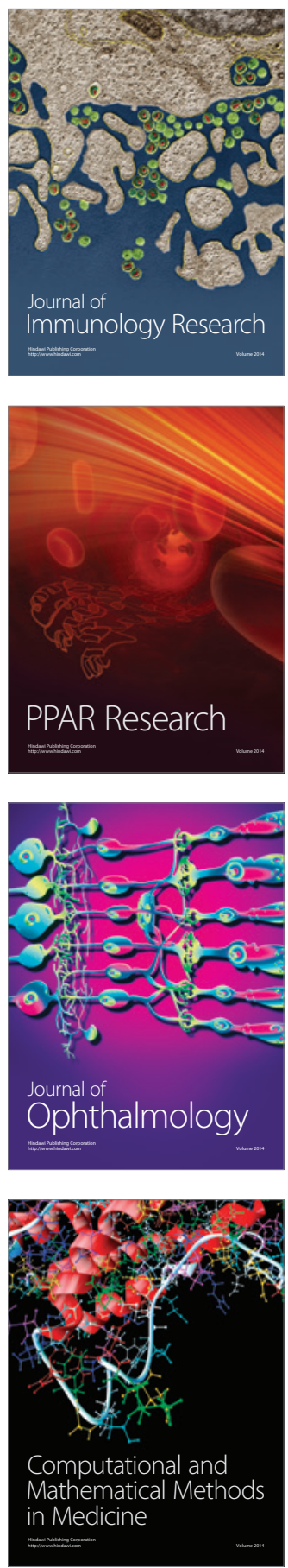

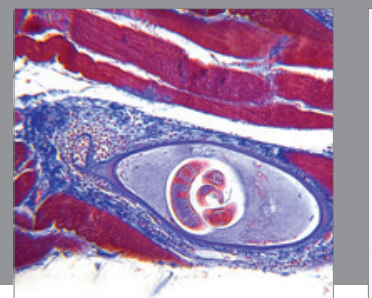

Gastroenterology

Research and Practice
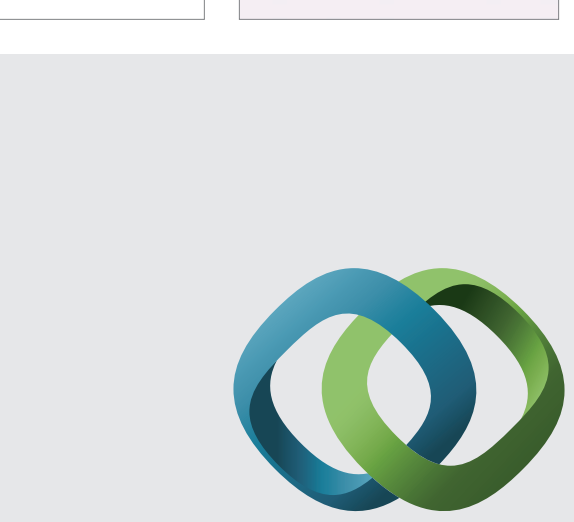

\section{Hindawi}

Submit your manuscripts at

http://www.hindawi.com
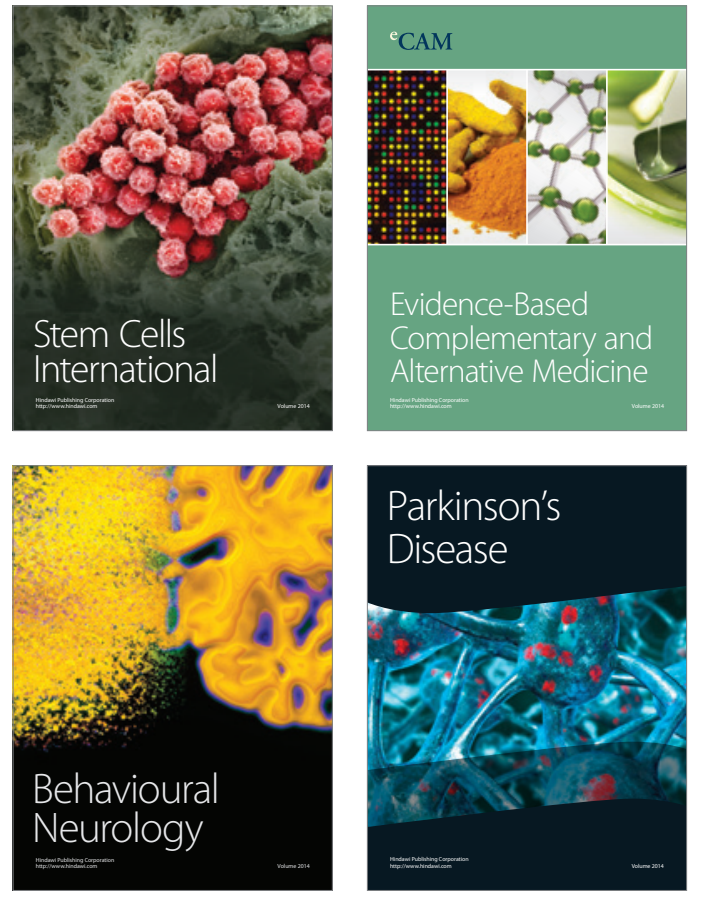
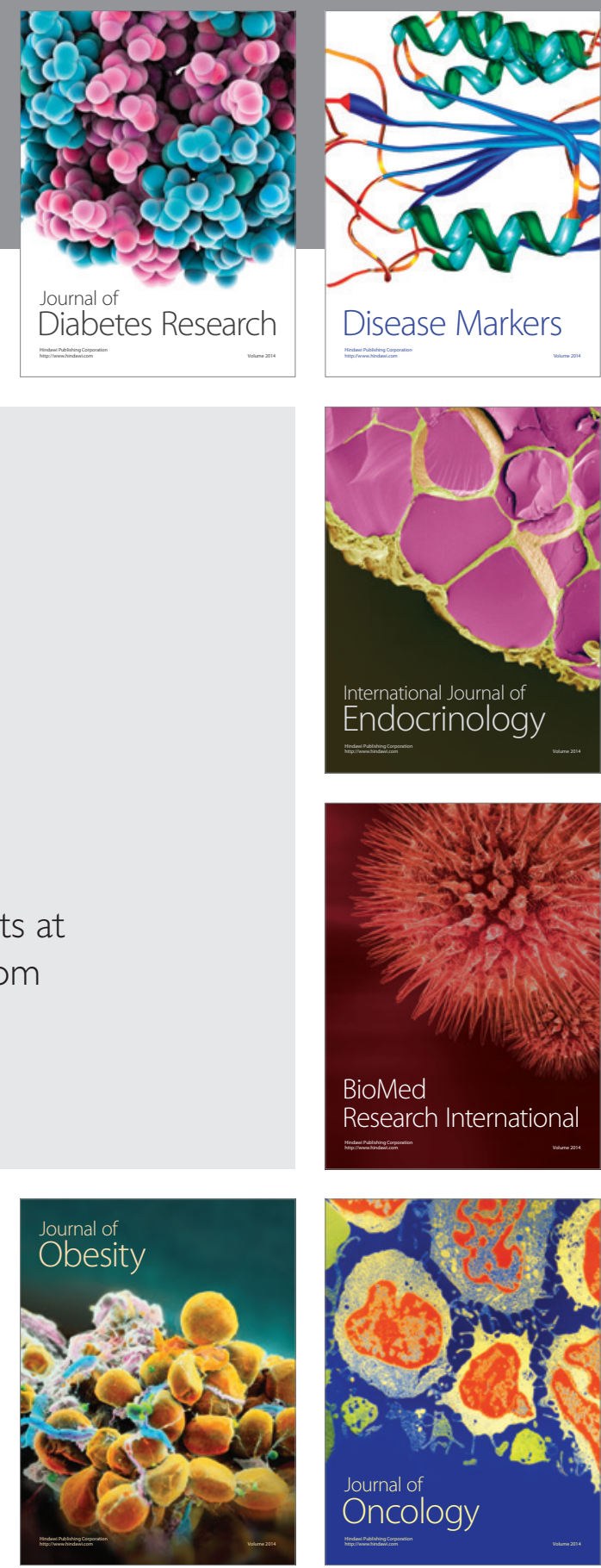

Disease Markers
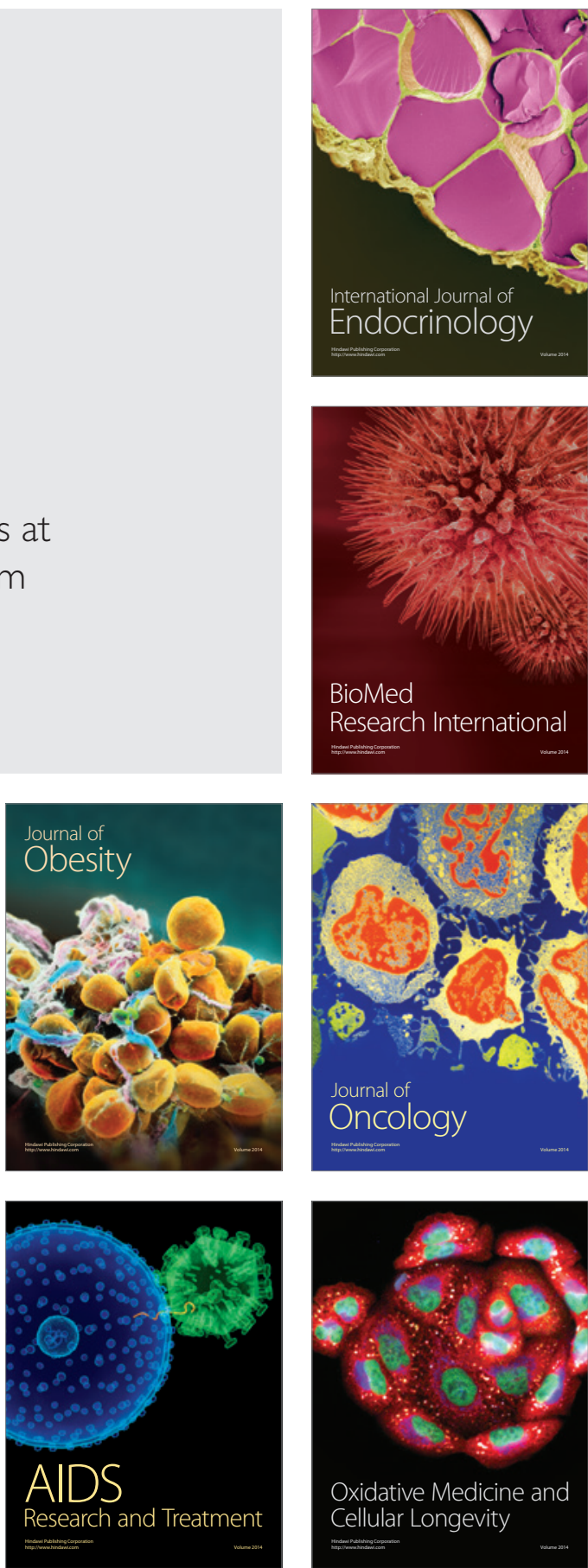